\title{
Dynamics of the dispersion interaction in an energy transfer system
}

\author{
David L. Andrews," David S. Bradshaw, Jamie M. Leeder and Justo Rodríguez
}

\author{
Received 28th February 2008, Accepted 30th May 2008 \\ First published as an Advance Article on the web 8th July 2008 \\ DOI: $10.1039 / b 803546 f$
}

On the propagation of resonant radiation through an optically dense system, photon capture is commonly followed by one or more near-field transfers of the resulting optical excitation. The process invokes secondary changes to the local electronic environment, shifting the electromagnetic interactions between participant chromophores and producing modified intermolecular forces. From the theory it emerges that energy transfer, when it occurs between chromophores with electronically dissimilar properties, can itself generate significant changes in the intermolecular potentials. This report highlights specific effects that can be anticipated when laser light propagates across an interface between differentially absorbing components in a model energy transfer system.

\section{Introduction}

Resonance energy transfer (RET), the transportation of electronic excitation between donor and acceptor units (ions, atoms, molecules or chromophores) following photoexcitation, is a mechanism of remarkable relevance across a wide range of physical, chemical and biological systems. One of its most widely studied manifestations is the process of energy hopping between chromophores within light-harvesting complexes-both natural ${ }^{1-5}$ and synthetic polymers such as dendrimers. ${ }^{3,6-9}$ In the latter connection we have recently reported the results of several calculations on energy flow ${ }^{10-12}$ and competing two-photon processes. ${ }^{13}$ Beyond the sphere of dendrimers, RET has been employed to determine a wide range of other polymeric properties; the 'spectroscopic ruler' has been readily utilised as a means of elucidating physical and morphological interface properties in complex polymer blends, for example. ${ }^{14,15}$ The well explored sensitivity of RET to transfer distance also proves invaluable in protein structure analysis, where it represents a powerful means of determining functional group separations and conformational dynamics. $^{3,16-19}$

It appears that little regard has been given to changes, intrinsic to the operation of RET, that occur in the dispersion interaction between donor and acceptor units. The dispersion interaction is itself most accurately described in terms of the Casimir-Polder potential; ${ }^{20}$ using quantum electrodynamics (QED), its explicit form emerges from calculations based on intermolecular coupling through virtual photon mediators. ${ }^{21}$ Recent work by Salam has determined the general formula for the dispersion potential deriving from multipolar interactions, ${ }^{22-24}$ also implementing quantum amplitude calculations using a state-sequence approach ${ }^{25}$ - a device first proposed and developed by Jenkins et al. ${ }^{26}$ Although the long-range behaviour of the leading contribution to the potential runs

Nanostructures and Photomolecular Systems, School of Chemical Sciences, University of East Anglia, Norwich, UK NR4 $7 T J$.

E-mail: david.andrews@physics.org with the inverse seventh power of the inter-particle distance $R$, the shorter-range form that operates over distances where effects are most pronounced exhibits an $R^{-6}$ asymptotic behaviour. The latter is well known as the attractive component of the Lennard-Jones potential. Although the dispersion potential is usually considered as an interaction between molecules in their ground states, a potential of similar form may readily be derived for molecules in excited states. ${ }^{27}$ Attention has recently been drawn to other forces, attributable to $\mathrm{RET},{ }^{28}$ and considered a consequence of interaction with the field emitted in donor decay. The latter potential is embedded in the Casimir-Polder potential for excited state molecules, emerging in the QED formulation as a 'contribution from real photons. ${ }^{29}$

Since the form of the dispersion interaction depends on the electronic states of the molecular participants, the dispersion force between neutral molecules is clearly subject to change during the course of absorption and RET. Indeed, electronic environments will first experience change upon local optical excitation of any donor, the associated modification of electromagnetic interactions between the donor and other units immediately producing modified intermolecular forces. In general, a degree of local movement can be expected as the system becomes accommodated to the new potential energy field. If the absorbed energy then transfers to a neighbouring acceptor unit of another species so that the latter acquires the excitation (i.e. RET occurs), the local electronic environment will suffer further change, and once again a compensating spatial accommodation can be expected to occur. In particular, in a solid-state environment where intermolecular forces are balanced in an equilibrium configuration, any changes associated with the migration of local electronic excitation should effectively act as a small perturbation to the equilibrium of intrinsic forces, producing measurable displacements.

This paper reports the results of calculations casting a new light on the physical link between these features. First, a succinct treatment of the dispersion pair potential is given, in which the dependence on the electronic state of the 
interacting particles is explicitly delivered. Since any adaptation to subtly changing force fields is most readily tested in an ensemble, rather than in individual particle pairs, the results are then applied to an experimentally meaningful system in which the two units between which energy is transferred are arrayed on parallel planes in close proximity. The theory is further developed to elicit the dynamical behaviour. Here the developing response of the system, following initial excitation by a realistically shaped pulse of laser light, is ascertained as a function of time. Possible developments of the theory are then discussed in the final conclusion.

\section{Dispersion interaction}

The dispersion potential is characterised by interactions between molecules in the short-range region, beyond significant orbital overlap. In the formal theory of QED, these interactions are considered to derive from an exchange of two virtual photons between the participant molecules. A variety of methods is available for the detailed calculations ${ }^{23,24,27}$ but in each case, in order to secure results applicable to arbitrarily large distances, $\dagger$ considerable complexity is entailed. For present purposes, however-where the focus is on interactions within the Förster radius, and therefore over distances where retardation effects are negligible - a simpler and much more direct method can be employed, as shown below.

Consider first a pairwise coupling between a donor $A$ and acceptor $B$, both having permanent electric dipoles. (This is to establish the formalism - in the main development of the dispersion interaction below, it will be assumed that the molecules involved are non-polar). In a QED derivation of the coupling, the interaction is represented as a virtual photon transfer between $A$ and $B$-one that conveys no energy (see Fig. 1). Within the near-field range, the donor emits a photon that is instantly absorbed by the acceptor, and the coupling can be considered unretarded. To determine a general expression for the corresponding interaction potential, since the virtual photon creation and annihilation events in effect occur simultaneously, the calculation can be treated by first-order perturbation theory using a pairwise operator $\boldsymbol{W}_{A B}$, given explicitly by;

$$
\boldsymbol{W}_{A B}=\frac{\mu_{i}^{A} \mu_{j}^{B}}{4 \pi \varepsilon_{0} R^{3}}\left(\delta_{i j}-3 \hat{R}_{i} \hat{R}_{j}\right) .
$$

Here, $\boldsymbol{\mu}^{\xi}$ is a dipole moment operator which operates on the states of molecule $\xi$, and $\boldsymbol{R}$ is the intermolecular separation vector; summation over repeated Cartesian indices is implied. The pair interaction potential is thus determined by $\Delta E=$ $\left\langle\Lambda\left|\boldsymbol{W}_{A B}\right| \Lambda\right\rangle$. In the latter, $|\Lambda\rangle$ signifies the unperturbed basis state involving the donor molecule in state $a$, the acceptor in state $b$ (plus the vacuum radiation field, i.e. no input radiation present), the product state representable as $|\Lambda\rangle=\left|A^{a} ; B^{b}\right\rangle$. Therefore, the interaction potential emerges from (1) with the diagonal matrix elements $\mu_{i}^{a a(A)} \equiv\left\langle A^{a}\left|\mu_{i}^{A}\right| A^{a}\right\rangle$ and $\mu_{j}^{b b(B)}$ (i.e. the static dipole moments) substituting for the dipole operators.

\footnotetext{
$\dagger$ Retardation effects come into play at intermolecular distances
} comparable with the principal absorption wavelengths.

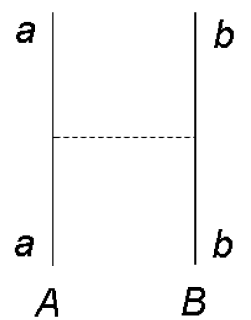

Fig. 1 Feynman diagram for the coupling between the permanent dipole of $A$ with that of $B$. Here, the donor and acceptor (world-lines on the left and right) are in the unperturbed states $a$ and $b$, respectively, and the dotted vertical line denotes virtual photon transfer.

The dispersion interaction is an additional form of coupling which, in the case of interaction between non-polar molecules, becomes the dominant form-his is the focus of the studies reported below. Fig. 2 illustrates a simplified Feynman diagram for evaluating the dispersion potential in its nearzone asymptote, where the coupling derives from the transfer of two virtual photons between $A$ and $B$. The coupling is treated, again through use of $\boldsymbol{W}_{A B}$, with secondperturbation theory, i.e.;

$$
\Delta E=\sum_{S} \frac{\left\langle\Lambda\left|\boldsymbol{W}_{A B}\right| S\right\rangle\left\langle S\left|\boldsymbol{W}_{A B}\right| \Lambda\right\rangle}{E_{\Lambda}-E_{S}},
$$

where $|S\rangle$ is an intermediate or virtual state of the system. On substitution of eqn (1) into (2), with the state of each component duly specified, the following emerges;

$$
\begin{aligned}
\Delta & E\left(A^{a} B^{b}\right) \\
= & \sum_{r, s} \frac{\left\langle A^{a} ; B^{b}\left|\boldsymbol{W}_{A B}\right| A^{r} ; B^{s}\right\rangle\left\langle A^{r} ; B^{s}\left|\boldsymbol{W}_{A B}\right| A^{a} ; B^{b}\right\rangle}{E_{a}^{A}+E_{b}^{B}-E_{r}^{A}-E_{s}^{B}} \\
= & \frac{1}{16 \pi^{2} \varepsilon 2_{0}^{2} R^{6}} \\
& \times\left[\frac{\mu_{i}^{a r(A)} \mu_{j}^{b s(B)} \mu_{k}^{r a(A)} \mu_{l}^{s b(B)}\left(\delta_{i j}-3 \hat{R}_{i} \hat{R}_{j}\right)\left(\delta_{k l}-3 \hat{R}_{k} \hat{R}_{l}\right)}{E_{a r}^{A}+E_{b s}^{B}}\right],
\end{aligned}
$$

with implied summation over repeated Cartesian (subscript) indices, summation also being effected over molecular states $\left|A^{r}\right\rangle$ and $\left|B^{S}\right\rangle$. Whilst (3) is generally valid for rigidly oriented molecules, the key features of the physics are clarified by

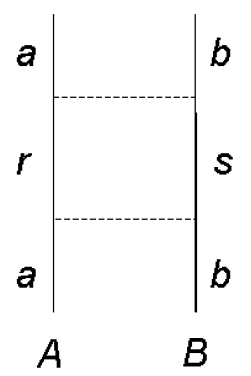

Fig. 2 Feynman diagram for the dispersion interaction between $A$ and $B$. Here, as in Fig. 1 but with inclusion of the intermediate states denoted by $r$ and $s$. 
performing an orientational average; as is assumed in the following model system calculations, the dipole moments of both the donor and acceptor are considered to be randomly oriented, in situ. With the isotropic average applied to the result emerging from eqn (3) the following is ascertained;

$$
\Delta E\left(A^{a} B^{b}\right)=-\frac{1}{24 \pi^{2} \varepsilon_{0}^{2} R^{6}} \sum_{r, s} \frac{\left|\boldsymbol{\mu}_{A}^{a r}\right|^{2}\left|\boldsymbol{\mu}_{B}^{b s}\right|^{2}}{E_{r a}^{A}+E_{s b}^{B}}
$$

which reduces to the well-known formula when $a$ and $b$ are ground levels. In the latter case each $E_{r a}^{A}$ and $E_{s b}^{B}$ is positive and, therefore, the result of eqn (4) is invariably a negative quantity. With due regard to the inverse power dependence on distance, the attractive nature of the dispersion potential is thus apparent. In the following, as an expedient but very good approximation, we shall assume that the state summations in eqn (4) are limited to the three states that determine the most prominent optical features. Thus, the donor and acceptor are chosen to have the significant molecular states with labels $r \in$ $\left\{0, \alpha, \alpha^{*}\right\}$ and $s \in\left\{0, \beta, \beta^{*}\right\}$. Of these, the states $\left|A^{\alpha^{*}}\right\rangle$ and $\left|B^{\beta^{*}}\right\rangle$ are specifically included as representatives of unpopulated molecular states.

\section{Model system}

Let us now consider a simple photophysical sequence of interactions engaging an interplay of the three-state molecules introduced in the last section. We consider a process that is initiated by the input of light of frequency, $\nu$, resonant to an absorptive transition in $A$, between its ground state and vibrational levels of an electronic excited state $\alpha$. The corresponding rate coefficient, $k_{\mathrm{abs}}$, has a time variation through its dependence on the temporal profile of the input - which in the study below is to be modelled by a Gaussian shape. The optical input is also assumed to be such that incident photons exceed the energy for $B$ to undergo transition to its first excited state $\beta$, but that their energy is insufficient to excite the higher states $\alpha^{*}$ or $\beta^{*}$; the latter are not populated under these conditions.

The processes that follow the initial absorption are summarised in Fig. 3. The first is internal vibrational relaxation (IVR), dissipating part of the energy acquired by $A$. The molecule can subsequently relax to the electronic ground state through a variety of direct or indirect mechanisms - spontaneous emission, RET, internal conversion, intersystem crossing, etc. For simplicity, all such electronic relaxation processes, with exception of RET, are included in a representative rate constant $k_{\text {rel }}^{\alpha}$. RET between $A$ and $B$ generally signifies that a downward transition occurs in $A$, to a vibrational level of the ground electronic state, while the released energy produces an upward transition in molecule $B$, from its ground state to a vibrational level of the electronic state $\beta$. The requirement for energy conservation is manifest in that the quantum amplitude for RET is proportional to a frequency-weighted overlap between the absorption spectrum of the acceptor and the emission spectrum of the donor, accommodating all possible routes for the energy relocation. Generally, the 'forward' process is efficiently achieved when there is a spectroscopic gradient in the direction of the transfer, $E_{\alpha 0}>E_{\beta 0}$, reducing significantly 'backward' RET; ${ }^{30}$ the low probability of the latter is signified by its neglect in the Figure and in subsequent calculations. The last stage of the process is the vibrational and electronic relaxation of $B$, following its excitation. Here, all relaxation processes are accommodated by a representative rate constant $k_{\text {rel. }}^{\beta}$.

Based on the principles outlined above, applicable to an isolated donor-acceptor pair, the dynamics associated with an ensemble is developed in the following section. Before pursuing the details, it is worth observing a simplifying feature that arises when more than one donor (or acceptor) is present. Consider, for example, the implications of having several chemically equivalent donors, in close proximity to a given donor $A$. In principle, RET among these molecules is also possible. However, the rapid IVR that follows initial donor excitation will generally put that molecule into an energy level where its decay has relatively small overlap with the red end of a neighbour's absorption profile. In the competing process of RET to a nearby acceptor, the associated spectroscopic gradient $^{30}$ will generally engender a much larger spectral overlap and hence a significantly larger transfer rate. For the same reason, it is possible to discount any formation of delocalised excitons, since that depends on the same form of coupling as RET between like chromophores. For the present we exclude the possibility of phonon-assisted relaxation, assuming the chromophore separations, or degree of disorder, are such that the process can be considered negligible in the chosen system. Markedly different behaviour would be anticipated in the case of $J$-aggregates. ${ }^{31}$

\section{Donor-acceptor ensemble}

With the model in place, the next step is to develop the kinetics for an ensemble composed of donors and acceptors, with $N$ molecules of each, counterpositioned across a gap set up in a vacuum or in air. To determine the average dispersion potential, $\overline{\Delta E}$, for this system, a summation over all the possible state permutations of $\Delta E\left(A^{a} B^{b}\right)$ is required. Overall there are four permutations to be taken into account, and from (4) the ensemble energy emerges as follows;

$$
\overline{\Delta E}=-\frac{1}{24 \pi^{2} \varepsilon_{0}^{2} R^{6}} \sum_{\substack{a=0, \alpha, b=0, \beta}} N_{a}^{\prime} N_{b}^{\prime \prime} \sum_{\substack{r=0, \alpha, \alpha^{*}, s=0, \beta, \beta^{*}}} \frac{\left|\boldsymbol{\mu}_{A}^{a r}\right|^{2}\left|\boldsymbol{\mu}_{B}^{b s}\right|^{2}}{E_{r a}^{A}+E_{s b}^{B}} .
$$

In the first summation on the right-hand side of eqn (5), $N_{a}{ }^{\prime}$ and $N_{b}{ }^{\prime \prime}$ are the fractional populations of $A$ in state $a$ and of $B$ in state $b$, respectively; the time-dependence of $\overline{\Delta E}$ will emerge from the temporal behaviour of these populations. By the rules of perturbation theory, the terms in which $a=r$ and $b=s$ are excluded in the second summations of eqn (5).

To determine the population dynamics the system is modelled as being initiated by a pulse of laser light. The population of state $\alpha$ is determined by the kinetics of excitation to, and decay from $\alpha$; as shown in Fig. 3 we have:

$$
\frac{\mathrm{d}}{\mathrm{d} t} N_{\alpha}^{\prime}=k_{\mathrm{IVR}}^{\alpha \dagger} N_{\alpha}^{\prime} \ddagger k_{\mathrm{rel}}^{\alpha} N_{\alpha}^{\prime}-k_{\mathrm{RET}} N_{\alpha}^{\prime}
$$

The population of $\alpha$ is dependent on $k_{\mathrm{IVR}}^{\alpha}$, given that $k_{\mathrm{IVR}}^{\alpha \ddagger} \gg k_{\mathrm{abs}}, N_{0}^{\prime A} \gg N_{\alpha}^{\prime}{ }^{\ddagger}$ (the latter condition also signifying 


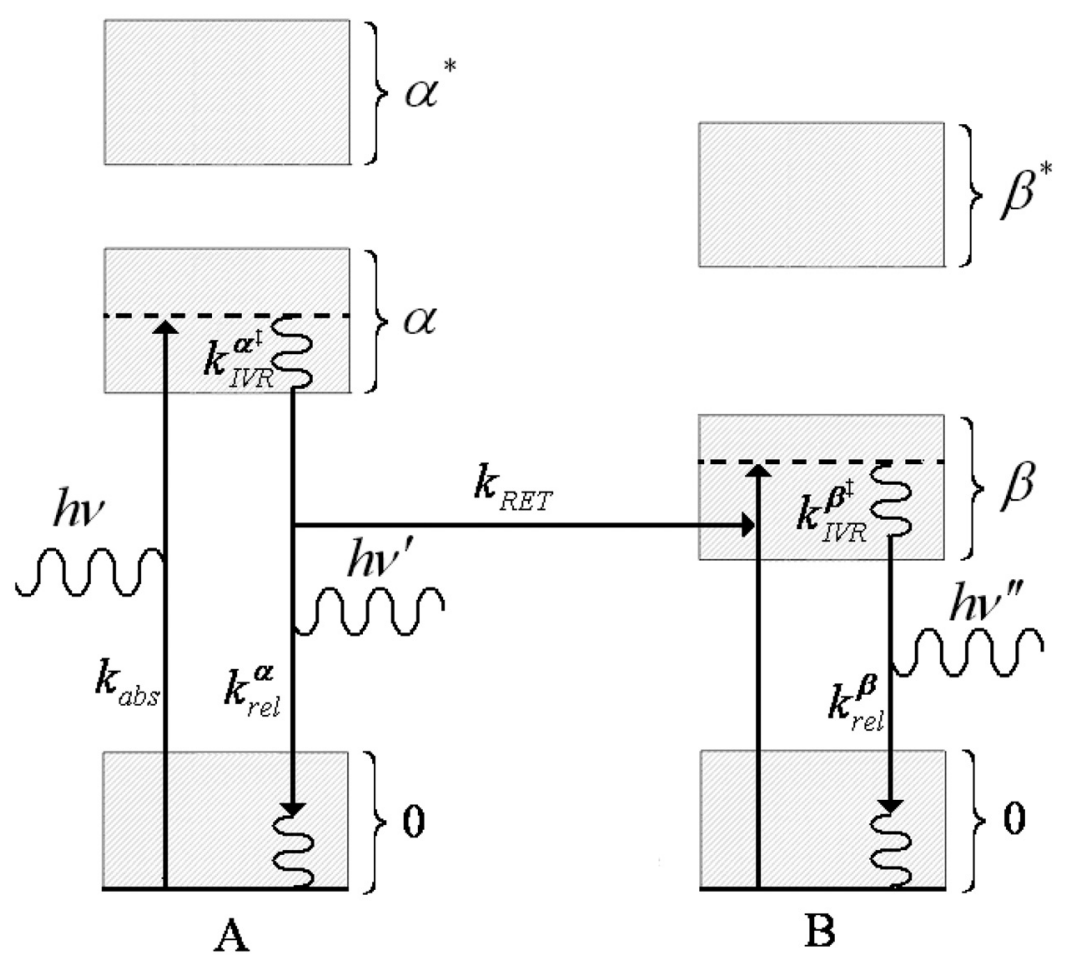

Fig. 3 Jablonski diagram, the rate constants $k$ denoting processes determining the excited populations of molecules $A$ and $B$. These processes involve the excitation of $A$ to a vibrational state of the electronic state $\alpha$ (with rate constant $k_{\text {abs }}$ ), internal vibrational relaxation (IVR) of $A$ ( $k_{\text {IVR }}^{\alpha}$ ), either spontaneous emission of photons $h \nu^{\prime}\left(k_{\mathrm{rel}}^{\alpha}\right)$ or RET exciting $B$ to an electronic state of $\beta\left(k_{\mathrm{RET}}\right)$, IVR of $B\left(k_{\mathrm{IVR}}^{\beta}\right)$, and spontaneous emission of photons $h \nu^{\prime \prime}\left(k_{\mathrm{rel}}^{\beta}\right)$. Each electronic relaxation is also typically accompanied by IVR as illustrated. See text for details.

that stimulated emission from $A$ is negligible) and the timescale for excitation to $\alpha^{\ddagger}$ is assumed to be short with respect to the laser pulse duration. It is possible to express (6) in the form of a simple, analytically solvable differential equation:

$$
\frac{\mathrm{d}}{\mathrm{d} t} N_{\alpha}^{\prime}=k_{\mathrm{abs}}-N_{\alpha}^{\prime}\left(k_{\mathrm{abs}}+k_{\mathrm{rel}}^{\alpha}+k_{\mathrm{RET}}\right) .
$$

Under the conditions $k_{\text {IVR }}^{\beta^{\ddagger}} \gg k_{\text {RET }}$ and $N_{\alpha}^{\prime} \gg N_{\beta}^{\prime \prime}{ }^{\ddagger}$, the ensuing growth in population of $\beta$ is seen to be dominated by $k_{\mathrm{RET}}$, and the following expression represents the time-dependent variation in $N_{\beta}{ }^{\prime \prime}$ :

$$
\frac{\mathrm{d}}{\mathrm{d} t} N_{\beta}^{\prime \prime}=k_{\mathrm{RET}} N_{\alpha}^{\prime}-k_{\mathrm{rel}}^{\beta} N_{\beta}^{\prime \prime}
$$

The constants of integration for both solutions (7) and (8) are determined by setting the physically reasonable initial condition that $N_{\alpha}{ }^{\prime}$ and $N_{\beta}{ }^{\prime \prime}$ are effectively zero at time zero; excluding excitation by intrinsic thermal energy, all molecules are in the ground state prior to delivery of the optical input.

The modelled fractional populations for $\alpha$ and $\beta$ are shown in Fig. 4, plotted for picosecond excitation. The maximum value of $N_{\alpha}{ }^{\prime}$ has been set by adjustment of the laser intensity profile maximum to a value of 0.5 . For this set of results, $k_{\mathrm{RET}}$ accounts for the majority of the total decay of $\alpha$, relative to $k_{\text {rel. }}^{\alpha}$. Such a condition represents a sufficiently strong shortrange interaction between $A$ and $B$, that RET is the dominant decay process for $\alpha$-as is always the case for donor-acceptor pairs within the Förster radius.

\section{Array assembly}

The last stage in the development of results for a meaningfully scaled system is to extend consideration to a structurally ordered implementation of the previous ensemble. This involves two parallel square-based arrays - one comprising donors and the other, acceptors-displaced by a distance $d$ and each composed of $N$ molecules (Fig. 5). Within each plane, these molecules are chemically identical and equally

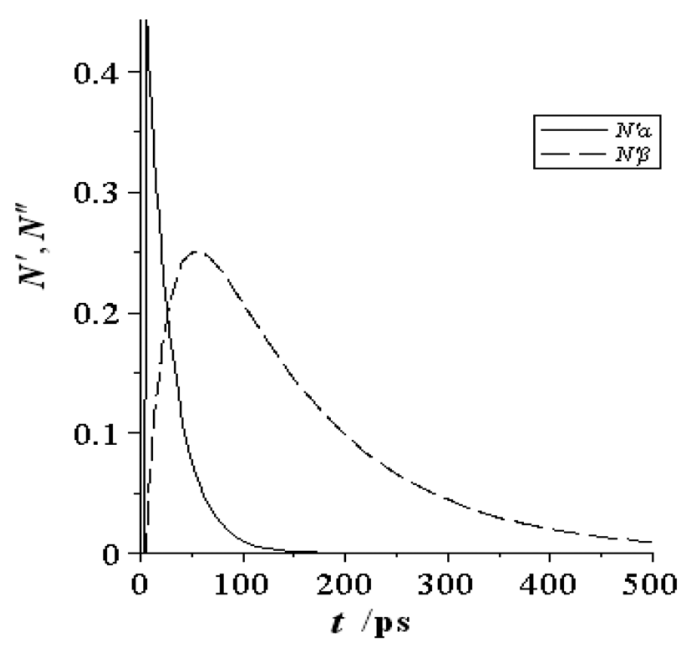

Fig. 4 Predicted $N_{\alpha}{ }^{\prime}$ and $N_{\beta}{ }^{\prime \prime}$ excited state population profiles following picosecond laser excitation. Values of $k_{\mathrm{RET}}$ and $k_{\mathrm{rel}}^{\alpha}$ are chosen such that $k_{\text {RET }}$ accounts for $80 \%$ of the total decay of $N_{\alpha}{ }^{\prime} ; k_{\text {rel }}^{\alpha}$ and $k_{\text {rel }}^{\beta}$ are taken as equal. 


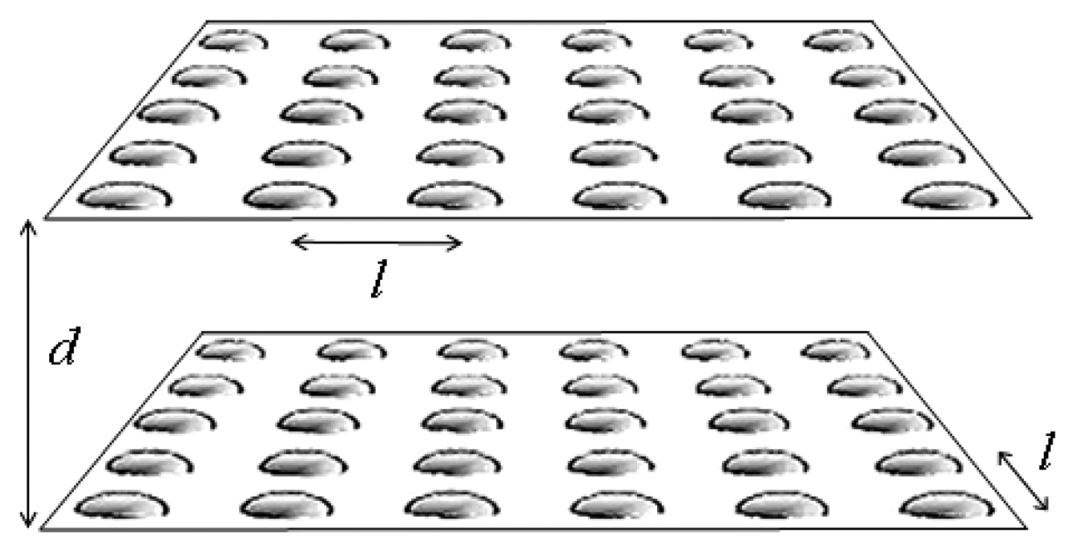

Fig. 5 Pair of parallel arrays displaced from each other by $d$. Each array is composed of identical molecules on a square lattice, a distance $l$ apart.

spaced from their nearest neighbours by a lattice constant, $l$; a difference in chemical composition of the donors and acceptors achieves the spectroscopic gradient that precludes backtransfer. Determining the dispersion potential per donor, $\overline{\Delta E}$, for the array system, based on the results for the time-evolving populations $N_{\alpha}{ }^{\prime}$ and $N_{b}{ }^{\prime \prime}$ found previously, the following is established;

$$
\begin{aligned}
\overline{\Delta E}= & -\frac{1}{24 \pi^{2} \varepsilon_{0}^{2} l^{6}} \sum_{\substack{a=0, \alpha, b=0, \beta}} N_{a}^{\prime} N_{b}^{\prime \prime} \sum_{\substack{r=0, \alpha, \alpha^{*}, s=0, \beta, \beta^{*}}} \frac{\left|\boldsymbol{\mu}_{A}^{a r}\right|^{2}\left|\boldsymbol{\mu}_{B}^{b s}\right|^{2}}{E_{r a}^{A}+E_{s b}^{B}} \\
& \times \int_{-\infty}^{\infty} \int_{-\infty}^{\infty} \frac{1}{\left(d^{\prime 2}+n^{2}+m^{2}\right)^{3}} \mathrm{~d} n \mathrm{~d} m \\
= & -\frac{1}{48 \pi \varepsilon_{0}^{2} l^{2} d^{4}} \sum_{\substack{a=0, \alpha, b=0, \beta}} N_{a}^{\prime} N_{b}^{\prime \prime} \sum_{\substack{r=0, \alpha, \alpha^{*}, s=0, \beta, \beta^{*}}} \frac{\left|\boldsymbol{\mu}_{A}^{a r}\right|^{2}\left|\boldsymbol{\mu}_{B}^{b s}\right|^{2}}{E_{r a}^{A}+E_{s b}^{B}},
\end{aligned}
$$

where $R^{2}=d^{2}+(n l)^{2}+(m l)^{2}$, with $n$ and $m$ integers. In addition, $d^{\prime}=d / l$ denotes the aspect ratio of the planar array, and the double integration over $n$ and $m$, treated as continuous variables, is a suitable approximation for summation over all molecules in the acceptor array - exhibiting the fact that eqn (9) describes the dispersion energy associated with each single donor in its interaction with the entire acceptor array (of

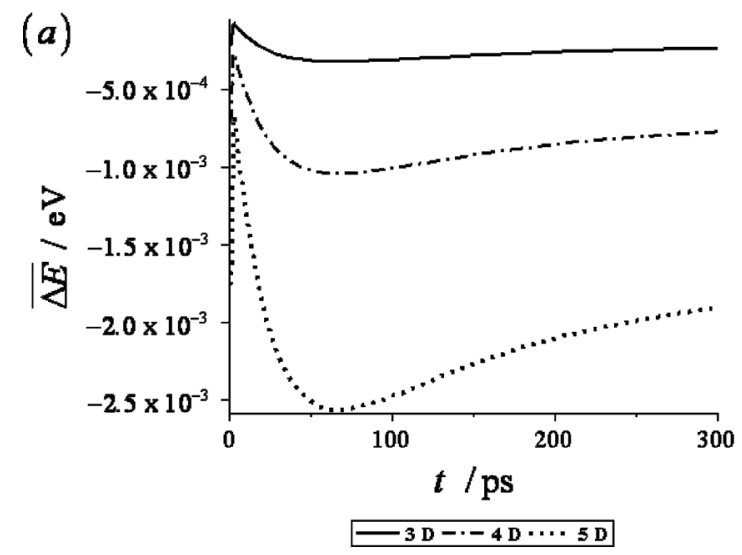

potentially infinite size although, in view of the distance dependence, generally dominated by a few close neighbours); it is not simply a summation over specifically correlated $A-B$ pairs.

From eqn (9), numerical results can be calculated to evaluate the physical consequences of electronic energy flow into, across, and out of the two arrays. Key determinants of the outcome are the magnitudes of the transition dipole moments $\boldsymbol{\mu}_{A}^{a r}$ and $\boldsymbol{\mu}_{B}^{b s}$, here for convenience set equal and ranging over modest values between 3 and $5 \mathrm{D}$. For ease of calculation, a figure of $0.8 \mathrm{~nm}$ is adopted for both $l$ and $d$. Transition energies to $\left|A^{\alpha^{*}}\right\rangle$ and $\left|A^{\alpha}\right\rangle$, from $\left|A^{0}\right\rangle$ are selected to correspond with wavelengths of 300 and $350 \mathrm{~nm}$, respectively. Transitions from $\left|B^{0}\right\rangle$ to $\left|B^{\beta^{*}}\right\rangle$ and $\left|B^{\beta}\right\rangle$ are lower in energy, wavelengths of 400 and $450 \mathrm{~nm}$ accordingly. A FWHM pulsewidth of 1.2 ps is assumed for the Gaussian profile input laser pulse.

Upon optical donor excitation, the initial increase in population of $\left|A^{\alpha}\right\rangle$ results in a corresponding increase in the potential energy of interaction between the donor and acceptor arrays. As illustrated in Fig. 6, there is a correspondingly rapid increase in the array interaction energy, reaching a maximum within 1-2 ps. The ensuing behaviour reflects the partial migration of electronic energy to acceptors, with the time-dependence of each excited state tempered by the various losses that lead to eventual decay. Over this interval,

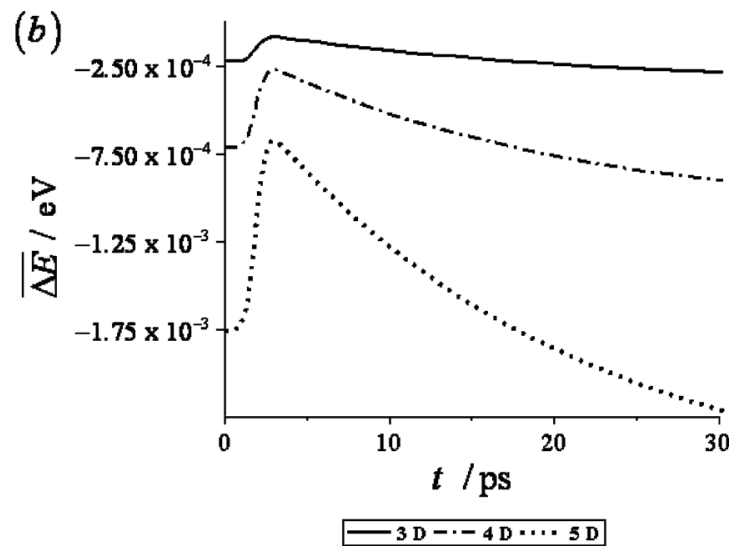

Fig. 6 (a) Evolution of the optically modified donor-acceptor interaction as a function of time, following pulsed laser excitation; (b) plot on magnified scale. 
compensating motions return the system to its original energy environment, as determined by the ground-state interaction potential. To quantify the corresponding ensemble-averaged forces involved, per donor, we develop eqn (9) into $\boldsymbol{F}_{\text {ave }}=$ $-(\partial \Delta \bar{E} / \partial d) \hat{\boldsymbol{n}}$, where $\hat{\boldsymbol{n}}$ is the normal to the plane of each array. For the range of $\overline{\Delta E}$ values presented in Fig. 6, the dispersion force for the donor-acceptor ensemble model varies in the picoNewton range. Taken as indicators of the feasibility of measurement, these figures are highly encouraging. With the rapidly ongoing development of techniques such as atomic force spectroscopy (AFM), ${ }^{32,33}$ such forces easily fall into the range of possible measurement.

\section{Conclusion}

Few areas of chemistry are not in some way linked with the underlying operation and influence of intermolecular forces. In the vast majority of these areas, where molecular matter generally resides in its electronic ground state, it is not surprising to find that the familiar forms of intermolecular potential are commonly adopted without necessary consideration of electronic state. Under conditions of thermal equilibrium, electronically excited state populations are usually vanishingly small, while even under the conditions that apply in active photochemical experimentation, the excited populations are, on the whole, severely limited in spatial and temporal extent. Nonetheless, it is incontrovertible that the nature of attraction between neutral molecules varies, even within those small regions of time and space, according to the electronic state.

This paper has represented a groundwork investigation into the modification of the dispersion force through RET. With a twofold aim it has sought to address, and to begin to quantify, the key issues. The first aim has been to characterise the changes to intermolecular force that occur on photoexcitation, using a robust quantum electrodynamical foundation. Secondly, by application to a simple model system, the analysis has aimed to illustrate the practicality of measuring the shifts in energy and force that must accompany photoexcitation in any multi-component system. In a system that displays typical resonance energy transfer behaviour, specific calculations based on an array configuration have exhibited a characteristic mechanical response and recovery, following the throughput of a resonant laser pulse. The effects are striking, and should prove amenable to measurement by currently available instrumentation.

Some issues invite further work to prompt and more accurately simulate experimentation. For example, at the price of considerable additional complexity, a more precise theoretical model might disengage the simplifying assumptions of ensemble average orientations, or the notionally perfect planarity of the donor and acceptor arrays. In comparison to the present predictions, based on isotropic averaging, the inclusion of orientational order in the calculations is expected to be reflected in an increase the mechanical response. Hence, it is likely to suggest a response that is more readily detectable than would appear from the possibly underestimated results reported here. It is our hope and intention that future work on these issues will play a role in developing and linking the theory into practical applications.

\section{Acknowledgements}

The authors are grateful to the EPSRC and the Leverhulme Trust for financing this work.

\section{References}

1 T. Markvart, Progr. Quant. Electron., 2000, 24, 107-186.

2 R. J. Cogdell, A. T. Gardiner, A. W. Roszak, C. J. Law, J. Southall and N. W. Isaacs, Photosynth. Res., 2004, 81, 207-214.

3 D. L. Andrews, Energy Harvesting Materials, World Scientific, Singapore, 2005.

4 A. N. Melkozernov and R. E. Blankenship, Photosynth. Res., 2005, 85, 33-50.

5 T. Polivka, R. G. Hiller and H. A. Frank, Arch. Biochem. Biophys., 2007, 458, 111-120.

6 A. Adronov and J. M. J. Frechet, Chem. Commun., 2000, $1701-1710$.

7 D. M. Guldi, Chem. Soc. Rev., 2002, 31, 22-36.

8 M. Cotlet, R. Gronheid, S. Habuchi, A. Stefan, A. Barbafina, K. Mullen, J. Hofkens and F. C. De Schryver, J. Am. Chem. Soc., 2003, 125, 13609-13617.

9 M. Takahashi, H. Morimoto, Y. Suzuki, T. Odagi, M. Yamashita and H. Kawai, Tetrahedron, 2004, 60, 11771-11781.

10 D. L. Andrews and D. S. Bradshaw, J. Chem. Phys., 2004, 121, 2445-2454.

11 D. L. Andrews and S. Li, Chem. Phys. Lett., 2006, 433, 239-243.

12 D. L. Andrews, S. Li, J. Rodríguez and J. Slota, J. Chem. Phys., 2007, 127, 134902.

13 D. S. Bradshaw and D. L. Andrews, Chem. Phys. Lett., 2006, 430, 191-194.

14 H. Morawetz, J. Polym. Sci., Part A: Polym. Chem., 1999, 37, 1725-1736.

15 J. P. S. Farinha and J. M. G. Martinho, in Fluorescence of Supermolecules, Polymers, and Nanosystems, ed. Berberan-Santos, Springer, Berlin, 2008, pp. 215-255.

16 C. G. Dos Remedios and P. D. J. Moens, in Resonance Energy Transfer, ed. D. L. Andrews and A. A. Demidov, Wiley, Chichester, 1999, pp. 1-64.

17 R. MacColl, Biochim. Biophys. Acta, 2004, 1657, 73-81.

18 K. E. Sapsford, L. Berti and I. L. Medintz, Angew. Chem., 2006, 45, 4562-4589.

19 R. R. Duncan, Biochem. Soc. Trans., 2006, 34, 679-682.

20 H. B. G. Casimir and D. Polder, Phys. Rev., 1948, 73, 360-372.

21 E. A. Power and T. Thirunamachandran, Phys. Rev. A: At., Mol., Opt. Phys., 1993, 48, 4761.

22 A. Salam, Int. J. Quantum Chem., 2005, 105, 762-766.

23 A. Salam, J. Phys. B, 2006, 39, S651-S662.

24 A. Salam, J. Phys. B, 2006, 39, S663-S670.

25 B. W. Alligood and A. Salam, Mol. Phys., 2007, 105, 395-404.

26 R. D. Jenkins, D. L. Andrews and L. C. D. Romero, J. Phys. B, 2002, 35, 445-468.

27 E. A. Power and T. Thirunamachandran, Phys. Rev. A: At., Mol., Opt. Phys., 1995, 51, 3660.

28 A. E. Cohen and S. Mukamel, J. Phys. Chem. A, 2003, 107, 3633-3638.

29 E. A. Power and T. Thirunamachandran, Chem. Phys., 1995, 198, 5.

30 D. L. Andrews and J. Rodríguez, J. Chem. Phys., 2007, 127, 084509.

31 C. Didraga, V. A. Malyshev and J. Knoester, J. Phys. Chem. B, 2006, 110, 18818-18827.

32 W. Baumgartner, P. Hinterdorfer and H. Schindler, Ultramicroscopy, 2000, 82, 85-95.

33 T. Hugel and M. Seitz, Macromol. Rapid Commun., 2001, 22, 989-1016. 